\title{
Arguments for and against HIV self-testing
}

This article was published in the following Dove Press journal:

HIVIAIDS - Research and Palliative Care

2 August 2014

Number of times this article has been viewed

\author{
Brian R Wood' \\ Carl Ballenger' \\ Joanne D Stekler ${ }^{1,2}$ \\ 'Division of Allergy and Infectious \\ Disease, University of Washington, \\ Seattle, WA, USA; 'Public Health, \\ Seattle and King County HIV/STD \\ Program, Seattle, WA, USA
}

\begin{abstract}
Approximately 60\% of human immunodeficiency virus (HIV)-infected individuals are unaware of their infection, and stigma and discrimination continue to threaten acceptance of HIV testing services worldwide. Self-testing for HIV has garnered controversy for years and the debate reignited with the approval of a point-of-care test for over-the-counter sale in the US in 2012. Here, we present arguments for and against HIV self-testing. The case in support of HIV self-testing contends that: the modality is highly acceptable, especially among the most at-risk individuals; self-testing empowers users, thus helping to normalize testing; and mutual partner testing has the potential to increase awareness of risk and avert condomless sex between discordant partners. Arguments against HIV self-testing include: cost limits access to those who need testing most; false-negative results, especially during the window period, may lead to false reassurance and could promote sex between discordant partners at the time of highest infectivity; opportunities for counseling, linkage to care, and diagnosis of other sexually transmitted infections may be missed; and self-testing leads to potential for coercion between partners. Research is needed to better define the risks of self-testing, especially as performance of the assays improves, and to delineate the benefits of programs designed to improve access to self-test kits, because this testing modality has numerous potential advantages and drawbacks.
\end{abstract}

Keywords: HIV, AIDS, self-testing, diagnosis, screening

\section{Introduction}

Globally, an estimated 60\% of human immunodeficiency virus (HIV)-infected individuals remain untested and unaware of their serostatus. ${ }^{1}$ Access to testing remains an issue in many regions and fear of stigmatization and discrimination continues to threaten acceptance of HIV testing services worldwide. ${ }^{2}$ In the US, approximately $20 \%$ of the more than one million persons living with HIV remain undiagnosed, and these individuals are responsible for nearly one-half of the new HIV transmissions in the country. ${ }^{3,4}$ Since 2006, the US Centers for Disease Control and Prevention have recommended routine HIV screening for patients between the ages of 13-64 years in all health care settings, as well as repeat testing at least annually for those at high risk for infection; however, the uptake of universal screening has been slow and hampered by numerous barriers. ${ }^{5,6}$ Accurate, widely acceptable, and easily accessible HIV screening methods are needed to increase the rates of diagnosis, allow for early antiretroviral therapy for the health of the infected individuals, and decrease the likelihood of transmission to susceptible persons. ${ }^{7}$

Proposals for HIV home tests generated controversy in the mid-1980s and debate reignited with the approval of a rapid oral swab kit for over-the-counter (OTC) sale in
Correspondence: Brian R Wood

Harborview 2W Clinic,

325 9th Avenue, Seattle,

WA 98104 USA

Tel +I 2064596410

Fax +l 2067445109

Email bwood2@uw.edu 
the US in $2012 .{ }^{8}$ On one hand, self-tests have the potential to reach persons who otherwise may never seek testing and are at highest risk for HIV infection, to empower users and help normalize screening, and to facilitate mutual partner testing and thus avert unprotected sex between discordant partners. On the other hand, cost and access, possible missed early infections due to the long window period, undiagnosed bacterial sexually transmitted infections (STIs), challenges to counseling and linkage to care, and the potential for coercion remain sharp criticisms to this mode of screening. Here, we review the history and performance of HIV self-tests and the arguments for and against this testing option (Figure 1).

\section{HIV self-testing: history and availability}

Self-testing for HIV involves collection of a saliva or finger stick blood sample by an individual, either in a supervised or unsupervised setting, for analysis by a laboratory or, more recently, for analysis by the user - much like a home pregnancy test. ${ }^{1,8}$ In general, individuals complete testing with little or no training. For unsupervised testing, written instructions and cautions are provided, as are toll-free telephone services for counseling and linkage to care.

Home tests for HIV are not novel. In 1996, the US Food and Drug Administration (FDA) approved the Home Access ${ }^{\circledR}$ HIV-1 Test System (Home Access Health, Hoffman Estates, IL, USA), a kit sold OTC that individuals could purchase and use anonymously at home. ${ }^{9}$ The test required prepaid mailing of a finger stick dried blood sample to a laboratory. Sensitivity and specificity of the test were estimated to be $>99.9 \%$, though warnings about missed infections in

Arguments for
- High acceptability, especially
among highest risk groups
- Results overall highly accurate
and trustworthy
- Empowers users and
decreases stigma
- Increases confidentiality and
privacy
- Promotes mutual partner
testing and may decrease rate
of condomless sex between
discordant partners

the window period were emphasized..$^{9,10}$ An advantage of the Home Access ${ }^{\circledR}$ Test was reflexive confirmation of positive results by the laboratory. A disadvantage was that users had to call to register before submitting their sample and then wait 7 days and call again to obtain results. ${ }^{9,11}$ Actual use never reached anticipated levels, primarily due to lack of awareness among at-risk individuals and concerns about accuracy and cost. ${ }^{12,13}$ However, in the first year of test availability, over 170,000 specimens were submitted: $95 \%$ were suitable for analysis; $0.9 \%$ were positive; $97 \%$ of users called to obtain results; and $58 \%$ reported they were testing for the first time. ${ }^{11}$

In 2002, the FDA approved the first point-of-care HIV test, the OraQuick Rapid HIV-1 Antibody Test (OraSure Technologies, Inc., Bethlehem, PA, USA) for use in health care settings in the US. ${ }^{14}$ This qualitative immunoassay used finger stick whole blood samples and provided results in 20 minutes. Sensitivity was reported to be $99.6 \%$ and specificity $100 \%{ }^{15}$ The largest advantage of the test was quick turnaround time for results, facilitating decision making in such settings as emergency rooms and labor and delivery units and in situations like occupational exposures. Again, warnings about false-negative results during the window period were frequent. With this test, cautions about the importance of confirmatory testing of positive results became prevalent. ${ }^{15}$

Over the coming decade, multiple additional point-ofcare HIV tests gained FDA approval for use in the US. ${ }^{8}$ This included rapid tests of whole blood, plasma, and oral fluid (using a swab of the gums), such as the OraQuick ADVANCE ${ }^{\circledR}$ Rapid HIV-1/2 Antibody Test (OraSure

Arguments against
- Longer window period than
lab-based screening tests
- Risk of false-negative results
and false reassurance during
acute infection
- Prohibitive cost and limited
access
- Insufficient counseling and
possible delayed entry to care
- Missed opportunities for STI
screening
- Potential for coercion

Figure I Arguments for and against the use of self-reporting HIV tests. Abbreviation: STI, sexually transmitted infection. 
Technologies, Inc.). For oral fluid, sensitivity of this assay was reported to be $99.3 \%$. In practice, studies of these point-ofcare tests showed that sampling of oral fluid identified fewer infections than plasma or whole blood and that point-of-care testing identified only $91 \%$ of antibody-positive men who have sex with men (MSM) and $80 \%$ of MSM detected by HIV RNA polymerase chain reaction. ${ }^{16,17}$ All antibody-based rapid assays are limited by a long window period, generally comparable to early-generation enzyme immunoassay tests and estimated to be up to $6-12$ weeks. ${ }^{16,18-20}$ Data indicated that individuals were more likely to receive results from rapid tests as compared to standard assays, though it was unclear whether this led to more frequent linkage to care. ${ }^{21-24}$ Additionally, although innovative programs began offering rapid testing through health fairs, door-to-door campaigns, mobile testing units, and other unique settings, all point-ofcare tests approved up to this time were available only to agents of clinical laboratories and approved only for use by health care personnel. ${ }^{1,8,25}$

It was not until 2012, 10 years after approval of the first rapid HIV assay, that the FDA approved a point-of-care test for home use. On July 3, 2012, the FDA approved the OraQuick oral swab for OTC sale to individuals 17 years or older. This test is now available at many pharmacies in the US. ${ }^{8,26}$ The FDA had set a prespecified criteria of $95 \%$ specificity and sensitivity to approve the test. Actual specificity was demonstrated to be $99.8 \%$ (indicating one in 5,000 results may be a false-positive), and actual sensitivity fell short of the target at $92 \%$ (indicating that one in 12 infections might be missed). However, the FDA constructed a Monte Carlo mathematical simulation model of self-test use that demonstrated that 4,000 infections might be prevented in the first year. ${ }^{8}$ The panel voted unanimously in favor of the test, ruling that the potential benefits outweighed the risks. ${ }^{26,27}$ Approval of the rapid kit for OTC sale recatalyzed debate about the benefits and dangers of self-testing. Whether this and other self-tests will have a significant impact on HIV around the globe remains to be seen. Self-tests remain illegal in some countries, though users may seek purchase on the Internet. ${ }^{8}$ There are numerous arguments both for and against HIV self-testing that must be considered by agencies considering approval of the tests and by users.

\section{Arguments for HIV self-testing: accessibility; accuracy; empowerment; and destigmatization}

An essential question is: "Will HIV self-tests be used and, if so, by whom?" Will these tests be utilized by the low-risk "worried well", or will they be accessed by the high-risk individuals who currently evade testing or need frequent repeat testing? ${ }^{28}$ In addition, how accurate are the results, and will users trust them? The preponderance of evidence demonstrates that rapid self-tests are accepted by those who are at the highest risk for HIV infection and that the results are highly accurate and trusted.

Two systematic literature reviews in 2013 analyzed the acceptability of HIV self-testing. ${ }^{1,2}$ The first reviewed data from eleven studies (two from sub-Saharan Africa, six from the US, two from Spain, and one from Singapore), nine of which used rapid testing (either blood or oral fluid-based). ${ }^{1}$ Overall, $70 \%$ of study participants accepted self-testing (range 22\%-87\%); acceptability was highest in a study from Malawi that involved home self-testing with minimal supervision and lowest in a study of health care workers from Kenya, though in this study acceptability increased to $97 \%$ in those who attended a pretest information session (and 55\% also took self-test kits for their partners). All studies in this review reported acceptability greater than $60 \%$ except one, and participants of the studies were mostly from high-risk groups (such as MSM, sex partners of high-risk MSM, and attendees of emergency departments or mobile testing units). In this review, overall $86 \%$ of self-test results agreed with results collected by health care staff; the rate of valid test results by users was $>97 \%$ in all studies but decreased to $92 \%$ in the study from Spain if the procedure was not demonstrated by trained staff beforehand and was only $46 \%$ in the study from Singapore in which users were required to transfer blood using a capillary tube. In this systematic review, no false-positive tests were reported, and false-negative results were rare and mostly due to misinterpretation of results. In the studies that reported rates of first-time users, as many as 50\% were testing for the first time, suggesting that self-testing attracted individuals who had never been tested by other means and were previously unaware of their serostatus.

The second systematic review evaluated supervised and unsupervised HIV self-testing in high- and low-risk populations. ${ }^{2}$ Twenty-one studies were included (16 in high-resource countries and five in resource-limited settings), giving an overall sample size of 7,512 participants in the unsupervised arm and 4,890 in the supervised arm. Acceptability was $74 \%-96 \%$ overall and $78 \%-84 \%$ in studies of unsupervised use. Additionally, 61\%-91\% of participants reported a preference for self-testing and $80 \%-97 \%$ reported partner self-testing. Specificity was observed to be $99.5 \%-100 \%$ for both strategies and sensitivity $92.9 \%-100 \%$ for unsupervised use and $97.5 \%-97.9 \%$ for supervised, though sensitivity data for unsupervised 
use was based on only one study. A recent analysis from Uganda further examined the accuracy of supervised versus unsupervised self-testing by randomizing 246 participants to supervised versus unsupervised oral swab self-testing..$^{29}$ The per-protocol analysis demonstrated a difference in sensitivity between the two strategies of $5.6 \%$ and noninferiority of unsupervised testing, though noninferiority was not shown in the intent-to-treat analysis. In this study, $75.4 \%$ of all users reported the test was "very easy" to use and they would recommend it to a friend or family member.

Several other studies have examined which individuals are likely to accept HIV self-testing. A cross-sectional telephone survey of adults (age 18-64 years) in New York City, NY, USA, in 2006 posed the question of whether respondents would use a rapid home test kit if available..$^{30} \mathrm{Of}$ the 6,639 adults who answered the survey, $56.2 \%$ reported they would use a test if available; participants from traditionally high-risk groups were more likely to report they would use a home test, such as blacks (70.7\%), Hispanics (73.3\%), persons age 18-24 (70.3\%), those with two or more recent sex partners (73.6\%), and those with recent MSM sexual activity $(67.6 \%)$. This analysis identified cost as a major barrier, which will be discussed later as a potential barrier to self-testing in the "Arguments Against" section.

A study in Seattle, WA, USA, randomized MSM to access to self-tests versus standard clinical tests and followed participants for a median of 10 months. ${ }^{31}$ In this trial, $96 \%$ in the self-testing arm reported that the kits were "very easy to use" and the remainder reported they were "somewhat easy to use". Of over 2,000 MSM who responded to an online survey in Australia in 2009, 67.4\% reported they would test more often if a rapid self-test were available. ${ }^{32}$ Independent predictors of increased testing frequency with home self-testing in this study included: preference for more convenient testing, not having to see a doctor when testing, wanting immediate results, and not being from an Anglo-Australian background among all men. In addition, recent condomless anal sex with casual partners was an independent predictor among previously tested men.

Multiple analyses have examined the acceptability of rapid self-testing in the emergency department, which is an important area in which to consider HIV testing options because emergency rooms attract large volumes of high-risk patients who often do not access regular primary care or other screening opportunities. Offering self-testing in this setting may increase testing rates by eliminating the need for staff to perform pointof-care testing, which reduces resources needed for testing and may be more acceptable to some patients. A study of adult emergency room patients in Baltimore, MD, USA showed that: $96.1 \%$ agreed to a self-point-of-care HIV test; $100 \%$ had concordant results with those of a trained provider; $91.7 \%$ "trusted the results very much"; and 98.4\% reported performing the test was "easy". ${ }^{33}$ An emergency department study that offered oral fluid self-tests and directions on tablet-based kiosks found that $49.5 \%$ accepted testing, and $100 \%$ of results were concordant between users and trained providers. ${ }^{34}$ Another study in Baltimore offered oral or finger stick self-testing to emergency room patients undergoing point-of-care oral fluid testing by a health care professional and showed that: $85 \%$ of participants consented to self-testing; $91 \%$ chose oral fluid over finger stick; $99.6 \%$ of self-test results agreed with health care professionals' results; and $84.4 \%$ of testers trusted their result. ${ }^{35}$ One consistent finding of self-testing studies across many settings is that self-tests of oral fluid are much more feasible and acceptable than those that require finger sticks. ${ }^{1}$

In addition to high acceptability and accuracy, an argument for HIV self-testing is the empowerment of individuals and the reduction of stigma, as well as the ability to test in an anonymous, confidential, and private manner. ${ }^{8}$ Stigma and discrimination remain huge barriers to HIV testing in health care settings, as are fear of confidentiality breaches and long wait times to receive results. ${ }^{2}$ In one of the systematic reviews from 2013, self-testing was perceived as, "highly confidential and private and participants believed self-testing could give people more power over their health". ${ }^{1}$ A study from Ethiopia showed that self-testing was highly acceptable among health care workers and the primary reason was increased privacy and confidentiality. ${ }^{36}$ Similarly, a program that offered confidential self-testing to health care workers in South Africa was accepted by $93 \%$ of candidates, and $91 \%$ reported a positive experience with testing. ${ }^{37}$

Another case for HIV self-tests is that they have the potential to increase awareness of risk, promote partner testing and disclosure, and possibly improve the accuracy of serosorting. For example, a group from New York provided a 3-month supply of HIV test kits to 27 ethnically diverse HIV-uninfected men who reported multiple male partners and little-to-no condom use for anal intercourse. A series of reports described outcomes among study participants. With access to self-testing kits, over $50 \%$ reported a change in their attitudes and/or behaviors, including a greater awareness of risk and more discussion of safe sex practices with partners. ${ }^{38}$ Participants also reported a change in choice of partners and in seven instances when a potential partner tested positive by a rapid HIV test kit, the sexual encounter stopped; more than one-half of the participants also ended sexual encounters when an HIV rapid test was refused by 
a sex partner. ${ }^{39}$ No sexual intercourse took place after a positive test, indicating that "point-of-sex" testing can lead to increased awareness of risk and can avert sex between discordant partners. ${ }^{40}$ In US-based studies, $73 \%$ of high-risk MSM (based on frequent condomless anal intercourse and changing sex partners) agreed to home self-testing, as did $82 \%$ of their sex partners. ${ }^{1}$ Another study of 60 participants who were interviewed before and after self-testing found that, "participants offered several possible strategies to introduce the home-test idea to partners, frequently endorsed mutual testing, and highlighted that home testing could stimulate greater honesty in serostatus disclosure". ${ }^{41}$ In this regard, HIV self-testing can facilitate mutual partner testing and can affect risk awareness and behavior.

Serosorting, the practice whereby an individual who believes himself or herself to be HIV-positive or negative attempts to only have sex with individuals he or she believes to be of concordant status, is controversial as an HIV prevention strategy. ${ }^{42}$ Data for the effectiveness of serosorting as an HIV prevention method are mixed. ${ }^{42-46}$ Limitations to serosorting include that individuals: may not be aware of their serostatus; may not be honest about their status; or may test negative but be in the window period. Self-testing could help those who are very high risk to be more aware of their status and could facilitate frequent testing and therefore may be of utility in this regard; however, concerns about missed infections and false reassurance in the window period abound. Whether self-testing will affect the accuracy of serosorting as an HIV prevention modality remains to be seen.

\section{Arguments against HIV self-testing: cost; missed early infections and bacterial STIs; challenges to counseling and linkage to care; and potential for coercion}

Although acceptability of rapid self-testing is high, access to self-tests remains a strong concern and multiple barriers persist. In particular, cost of tests is likely prohibitive to many individuals. For instance, in the 2006 telephone survey of New York City adults, although the highest-risk groups seemed most interested, it was estimated that over $50 \%$ of those who said they would use the test would not be able to afford it. ${ }^{30}$ Similarly, in Spain, only $17.9 \%$ of survey respondents said they would pay the US price for the test, and it was estimated that the test would not have a significant effect unless made more affordable. ${ }^{47}$ In a study that used conjoint analysis to examine willingness to test of at-risk individuals across many scenarios, the price of HIV self-testing (free versus \$50) had the most significant impact on willingness to test. ${ }^{48}$ In the randomized trial of self-tests versus clinic tests for MSM in Seattle, $46 \%$ reported the most they would pay for a home self-test would be $\$ 20$ or less, $26 \%$ would pay $\$ 20-\$ 40,17 \%$ would pay $\$ 40$ or more, and $11 \%$ would only use one if free (current cost of the test in the US is approximately \$40). ${ }^{31}$ The frequency with which participants in this trial expected to test varied depending on cost. Similarly, in a trial in Philadelphia, PA, USA, in which $91 \%$ of participants were willing to self-test, only $26 \%$ were willing to pay more than \$20 and only $14 \%$ were willing to pay the current US price, ${ }^{49}$ and in one of the emergency department self-testing trials in Baltimore, $35 \%$ of participants said they wouldn't pay more than $\$ 10$ for a self-test. ${ }^{33}$ Therefore, cost is a significant barrier to self-testing and will likely limit influence of the test if not addressed.

Access to self-tests, even in regions where legal, is a concern. An analysis that looked at the availability of selftest kits in pharmacies in New York City 1 year after FDA approval found that the kits were actually available in only $27 \%$ of pharmacies (24\% in high-morbidity neighborhoods and $30 \%$ in low-morbidity neighborhoods). ${ }^{50}$ Kits were more likely to be kept behind the counter and thus require interaction with the pharmacy staff in high-morbidity areas, and a majority of the pharmacies $(66 \%)$ set a price for the kits above the manufacturer's suggested retail price. Therefore, beyond legality and cost, awareness of the kits and access to the kits are continued obstacles.

Another significant concern is false-negative results or missed infections in the window period, especially because these early infections often exhibit high viral loads and elevated transmissibility. If individuals with frequent HIV risk behavior are accessing the test, which is the goal, a negative test during the window period may lead to false reassurance, which may increase condomless sex with HIV-negative partners and thus propagate transmission. ${ }^{8}$ This "risk compensation" has potentially dire consequences. For example, a modeling study that simulated what would happen if all MSM replaced clinic-based testing with home-use tests showed that HIV prevalence would increase from $18.6 \%$ to $27.5 \%$ if home use did not impact testing frequency and to $22.4 \%$ if home use increased testing frequency threefold. $^{51}$ This surge in HIV prevalence is secondary to increased HIV transmission during primary infection, the long window period of self-tests as compared to polymerase chain reaction and antigen-antibody based tests, and lower rates of linkage to care. This modeling analysis showed that 
any replacement of clinic-based testing with home-based testing would lead to an increased prevalence and suggested that achieving a reduced prevalence would require tests with a shorter window period plus increased testing frequency.

Because of the risk of missed early infections, materials that accompany self-test kits stress to users the risk of falsenegative results during the window period as well as signs of acute retroviral syndrome, and it has been emphasized that providers should not consider self-tests to be a replacement for clinic-based testing per national guidelines. ${ }^{28,52}$ Instead, self-tests should be considered a supplement or an alternative for patients who are not screening in health care settings. Of note, in the modeling study that simulated substitution of clinic-based tests with self-tests, if the self-tests did not replace clinical testing but instead were used as supplemental screening, prevalence decreased. ${ }^{51}$ Still, all positive self-test results must be confirmed and, in the setting of recent highrisk activity or symptoms of acute retroviral syndrome, all negative results should be repeated or ideally supplanted by a more sensitive test, such as an HIV ribonucleic acid assay, if available. ${ }^{28}$ Rapid assays used for point-of-care testing in health care settings have the same limitation of assays used for home self-testing. ${ }^{8}$ The issue becomes how patients will be counseled about these limitations, particularly with unsupervised self-testing.

The lack of formal or in-person counseling is a frequent argument against unsupervised HIV self-testing. Early versions of the home testing kit were banned in some countries due to the concern that false-negative tests would lead to false reassurance and that positive results would lead to suicidality and other adverse events. ${ }^{53}$ Concerns over "inaccuracy and risks of suicide" have surrounded self-tests since the mid-1980s. ${ }^{8,54}$ There is concern that without in-person counseling, patients miss opportunities to discuss risk prevention with their provider and may not be able to ask sufficient questions. There is also concern about psychological reactions when receiving a positive result in isolation. With the availability and effectiveness of antiretroviral therapy, suicidal thinking after a positive HIV test has become less frequent, but it still may occur and may be more likely in resource-limited settings. ${ }^{8}$

As an example of the dangers of a lack of formal counseling with self-testing, one case report describes a homeless Latino MSM who tested positive using a diverted rapid test kit from a self-testing trial. ${ }^{55}$ The test kit was given to him to use after a sexual encounter, and the result was positive. The individual assumed the result to be sufficient and did not understand the need for confirmatory testing and did not seek posttest counseling. He thus took 2 months to present to care. In addition, the partner who had supplied the kit did not seek postexposure prophylaxis. This case suggests that sex partners will indeed use the tests, and the individual who tested positive did report that he started using condoms with partners after receiving the result, but it also shows that not all will successfully utilize counseling hotlines. It also demonstrates some of the risks of self-testing.

Few studies have looked at actual rates of accessing phone counseling services or the effectiveness of phone counseling with HIV self-testing. However, one of the systematic reviews from 2013 identified five studies (three from the US and two from sub-Saharan Africa) that discussed this issue. ${ }^{1}$ Access to phone counseling was variable between studies but markedly lower in studies from Africa. African participants also voiced a stronger desire for comprehensive face-to-face counseling. A large majority of participants from the US (95\%) expressed a preference for posttest counseling by phone. A recent survey of university students from Canada and South Africa found that the majority preferred to receive STI results and counseling in person as opposed to via the internet or mobile phones, though this was not specific to HIV testing. ${ }^{56}$ The importance of face-to-face counseling may vary depending on geographic region, culture, and other factors; this is an area where further research is needed.

In addition to limited counseling, a disadvantage of HIV self-testing, particularly unsupervised, is how patients will be linked to care. If individuals are testing at home for fear of stigma and discrimination, they may also choose to avoid linking or presenting to care for the same fears. As in the case report described previously, patients may test and discover a positive result and then never call for support. ${ }^{55}$ In one review, $96 \%(102 / 106)$ of individuals self-testing for HIV said they would seek posttest counseling, but it is unknown how many actually did and data for actual linkage rates after self-testing are sparse. ${ }^{2}$ In the trial of self-testing from Philadelphia, PA, USA, 96\% reported they would seek medical care if they tested HIV-positive, but prospective data on the actual rates of linkage to care are needed. ${ }^{49}$ Studies have also shown that individuals who receive rapid HIV test results are less likely to return for results of STI testing, so increased rates of HIV self-testing may lead to missed opportunities for STI counseling and screening or linkage to STI services. ${ }^{57}$

An additional criticism of self-testing is the potential for gender-based or partner-based violence. In a communitybased self-testing program in Malawi, in which HIV self-testing had a high degree of acceptability and accuracy (76\% of 16,660 individuals successfully self-tested in the 
first year, 98\% said they would recommend a self-test, and agreement between self-tests and lab tests was 99.3\%) a concern elicited by surveys of participants was that of coercion. ${ }^{58}$ Out of 10,007 individuals surveyed, 287 reported being "forced to test" (2.3\% of women and 3.3\% of men). Though no serious cases of gender-based violence were reported, there is the potential that tests could lead to such instances or that the results could lead to partner-based violence. The issue of potential coercion must be weighed when considering the risks and benefits of self-testing.

Further disadvantages to self-testing exist in resourcelimited settings, such as issues with regulation of test development and sale, quality control of self-tests and storage of tests at acceptable temperatures, as well as heightened barriers to counseling and linkage to care given geographic distances and isolation. ${ }^{8}$ In addition, rapid tests, because they are antibody-based, have limited utility for HIV screening of infants.

\section{Conclusion and future directions}

In summary, the approval of a point-of-care assay for HIV self-testing by the FDA has significant implications for screening and public health efforts in the US and has raised issues that must be considered by other countries when considering approval of similar tests. Self-tests may reach the highest-risk individuals who currently are not being screened for HIV, may promote mutual partner testing and thus avert condomless sex between discordant partners, and may have the potential to decrease stigma and help normalize testing. However, numerous concerns persist, such as cost and access, possibility for false reassurance and missed early infections in the window period, as well as limited counseling and linkage to care options and the potential for coercion.

Recently, the FDA approved a rapid finger stick assay called the Determine ${ }^{\mathrm{TM}} \mathrm{HIV}-1 / 2 \mathrm{Ag} / \mathrm{Ab}$ Combo Test (Alere Inc., Waltham, MA, USA) for use in health care settings. ${ }^{59}$ This test combines a p24 antigen assay with traditional antibody testing, thus reducing the window period, though this point-of-care assay does not perform as well as lab-based fourth generation antigen-antibody combination tests. ${ }^{16,60}$ A street-based testing program in Spain demonstrated selftesting with this kit to be feasible, with $92 \%$ of participants obtaining a valid result. ${ }^{61}$ Would the window period of this test be short enough that the use for self-testing might help to decrease HIV prevalence? How short would the window period need to be for fears of false reassurance to be allayed? In the modeling study of Seattle MSM, a self-test with a window period that was shortened from 90 to 42 days led to decreased HIV prevalence if testing frequency also increased 1.6 fold. ${ }^{51}$ Thus, a point-of-care test like Determine ${ }^{\mathrm{TM}}$, which detects HIV 7-15.5 days earlier than the Western blot (earlier than the FDA-approved third generation but later than fourth generation enzyme immunoassay tests) and may allow for differentiation between p24 antigen and HIV-1/2 antibody positivity, is a step in the right direction, but these questions will require more discussion and more research. ${ }^{62}$

Other recent or forthcoming changes in the field of HIV self-testing have potential to impact the issue greatly. For instance, Chembio Diagnostics Systems, Inc., (Medford, NY, USA) recently received approval in Europe for the commercial sale of its SureCheck ${ }^{\circledR}$ Rapid HIV Test and started the process required for the submission of investigational device exemption to the FDA, which may lead to the approval of this test for OTC sale in the US. ${ }^{63,64} \mathrm{An}$ additional approved point-of-care test available to consumers may help with cost and access. Additionally, programs to help with the cost of self-tests are under way, such as a voucher program in Los Angeles County (CA, USA), which was recently shown to be feasible. ${ }^{65}$ Dissemination of such programs is needed so that individuals who would benefit from testing are not excluded due to cost. Novel programs, such as selling self-tests in vending machines, are being evaluated, ${ }^{66}$ and programs that offer self-test kits to newly diagnosed individuals for home testing of partners are being explored. New rapid assays that simultaneously detect HIV and syphilis could quell concerns that HIV self-testing will lead to missed STI treatment opportunities and propagation of syphilis. ${ }^{67}$

With these rapid changes to the landscape of HIV self-testing, many questions remain and further study is needed. Most importantly, more data on linkage to care and the risks of self-testing are crucial so that the dangers of self-testing can be addressed and this testing modality can be applied to large public health campaigns in a safe manner. Unique barriers to self-testing among specific groups, such as MSM, heterosexuals, or high-risk women should be explored, and factors influencing self-testing uptake as well as the optimal means for counseling and linkage to care in various regions of the world should be examined. Whether mobile technology can facilitate self-testing and improve counseling and linkage to care also deserves attention. Additionally, further data is needed regarding the advantages and disadvantages to self-testing for poor, marginalized ethnic minorities, who are disproportionately affected by HIV in many countries. The potential of selftesting to empower individuals and normalize screening 
should not be ignored. Hopefully, as test performance of the assays improves, additional assays become commercially available and drive down costs, and disadvantages of self-tests can be addressed, this strategy will offer a step toward averting the nearly 2.7 million new HIV infections that occur per year globally. ${ }^{8}$

\section{Disclosure}

The authors report no conflicts of interest in this work.

\section{References}

1. Krause J, Subklew-Sehume F, Kenyon C, Colebunders R. Acceptability of HIV self-testing: a systematic literature review. BMC Public Health. 2013;13:735.

2. Pant Pai N, Sharma J, Shivkumar S, et al. Supervised and unsupervised self-testing for HIV in high- and low-risk populations: a systematic review. PLoS Med. 2013;10(4):e1001414.

3. Campsmith ML, Rhodes PH, Hall HI, Green TA. Undiagnosed HIV prevalence among adults and adolescents in the United States at the end of 2006. J Acquir Immune Defic Syndr. 2010;53(5):619-624.

4. Marks G, Crepaz N, Janssen RS. Estimating sexual transmission of HIV from persons aware and unaware that they are infected with the virus in the USA. AIDS. 2006;20(10):1447-1450.

5. Branson BM, Hansfield HH, Lampe MA, et al; Centers for Disease Control and Prevention (CDC). Revised recommendations for HIV testing of adults, adolescents, and pregnant women in health-care settings. MMWR Recomm Rep. 2006;55(RR-14):1-17; quiz CE1-4.

6. Burke RC, Sepkowitz KA, Bernstein KT, et al. Why don't physicians test for HIV? A review of the US literature. AIDS. 2007;21(12): 1617-1624.

7. Cohen MS, Chen YQ, McCauley M, et al; HPTN 052 Study Team. Prevention of HIV-1 infection with early antiretroviral therapy. $N$ Engl J Med. 2011;365(6):493-505.

8. Myers JE, El-Sadr WM, Zerbe A, Branson BM. Rapid HIV selftesting: long in coming but opportunities beckon. AIDS. 2013;27(11): $1687-1695$.

9. US Food and Drug Administration [homepage on the Internet]. Information regarding the Home Access HIV-1 test system; 2012. Available from: http://www.fda.gov/BiologicsBloodVaccines/ BloodBloodProducts/ApprovedProducts/PremarketApprovalsPMAs/ ucm311903.htm. Accessed February 24, 2014.

10. Roberts J. US approves HIV "home test". BMJ. 1996;312(7042): 1317.

11. Branson BM. Home sample collection tests for HIV infection. JAMA. 1998;280(19):1699-1701.

12. Colfax GN, Lehman JS, Bindman AB, et al; Multisite Evaluation of Surveillance of HIV (MESH) Study Group. What happened to home HIV test collection kits? Intent to use kits, actual use, and barriers to use among persons at risk for HIV infection. AIDS Care. 2002;14(5): 675-682.

13. Spielberg F, Critchlow C, Vittinghoff E, et al; HIV Early Detection Study Group. Slow diffusion of home HIV-specimen collection: provider concerns at odds with client preferences. Sex Transm Dis. 2001;28(1):51-57.

14. US Centers for Disease Control and Prevention [homepage on the Internet]. Notice to Readers: Approval of a New Rapid Test for HIV Antibody; 2002. Available from: http://www.cdc.gov/mmwr/preview/ mmwrhtml/mm5146a5.htm. Accessed February 24, 2014.

15. US Food and Drug Administration. OraQuick Rapid HIV-1 Antibody Test. OraSure Technologies Inc., Bethlehem, PA, USA; 2003. Available from: http://www.fda.gov/downloads/BiologicsBloodVaccines/ BloodBloodProducts/ApprovedProducts/PremarketApprovalsPMAs/ UCM091989.pdf. Accessed February 24, 2014.
16. Stekler JD, O'Neal JD, Lane A, et al. Relative accuracy of serum, whole blood, and oral fluid HIV tests among Seattle men who have sex with men. J Clin Virol. 2013;58 Suppl 1:e119-e122.

17. Stekler JD, Swenson PD, Coombs RW, et al. HIV testing in a high-incidence population: is antibody testing alone good enough? Clin Infect Dis. 2009;49(3):444-453.

18. Masciotra S, McDougal JS, Feldman J, Sprinkle P, Wesolowski L, Owen SM. Evaluation of an alternative HIV diagnostic algorithm using specimens from seroconversion panels and persons with established HIV infections. J Clin Virol. 2011;52 Suppl 1:S17-S22.

19. Owen SM, Yang C, Spira T, et al. Alternative algorithms for human immunodeficiency virus infection diagnosis using tests that are licensed in the United States. J Clin Microbiol. 2008;46(5):1588-1595.

20. Cohen MS, Gay CL, Busch MP, Hecht FM. The detection of acute HIV infection. J Infect Dis. 2010;202 Suppl 2:S270-S277.

21. Delaney K, Knoble T, Rurangirwa J, et al. Using a Rapid HIV Testing Algorithm to Improve the Accuracy of HIV Testing, Receipt of Test Results, and Linkage to Care: Results of a Demonstration Project in 2 US Cities. In: Program and abstracts of the 18th Conference on Retroviruses and Opportunistic Infections (CROI); February 27-March 2, 2011; Boston, MA. Abstract \#132LB.

22. Keller S, Jones J, Erbelding E. Choice of Rapid HIV testing and entrance into care in Baltimore City sexually transmitted infections clinics. AIDS Patient Care STDS. 2011;25(4):237-243.

23. Hutchinson AB, Branson BM, Kim A, Farnham PG. A meta-analysis of the effectiveness of alternative HIV counseling and testing methods to increase knowledge of HIV status. AIDS. 2006;20(12):1597-1604.

24. Spielberg F, Branson BM, Goldbaum GM, et al. Choosing HIV Counseling and Testing Strategies for Outreach Settings: A Randomized Trial. J Acquir Immune Defic Syndr. 2005;38(3):348-355.

25. de la Fuente L, Delgado J, Hoyos J, et al; Madrid Rapid HIV Testing Group. Increasing early diagnosis of HIV through rapid testing in a street outreach program in Spain. AIDS Patient Care STDS. 2009;23(8): 625-629.

26. US Food and Drug Administration. FDA approves first over-the-counter home-use rapid HIV test [press release]. Silver Spring, MD: US Food and Drug Administration; July 3, 2012. Available from: http://www. fda.gov/NewsEvents/Newsroom/PressAnnouncements/ucm310542. htm. Accessed February 24, 2014.

27. Roehr B. FDA approves "instant" HIV home test. BMJ. 2012;345: e4636.

28. Paltiel AD, Walensky RP. Home HIV testing: good news but not a game changer. Ann Intern Med. 2012;157(10):744-746.

29. Asiimwe S, Oloya J, Song X, Whalen CC. Accuracy of Un-supervised Versus Provider-Supervised Self-administered HIV Testing in Uganda: A Randomized Implementation Trial. AIDS Behav. Epub April 2, 2014.

30. Myers JE, Bodach S, Cutler BH, Shepard CW. Acceptability for home tests kits for HIV in New York City, 2006. In: Program and abstracts of IDWeek 2012; October 17-21, 2012; San Diego, CA; Abstract 121.

31. Katz DA, Golden MR, Hughes JP, Farquhar C, Stekler JD. Acceptability and Ease of Use of Home Self-Testing for HIV among Men Who Have Sex with Men. In: Program and abstracts of 19th Conference on Retroviruses and Opportunistic Infections (CROI); March 5-8, 2012; Seattle, WA; Abstract 1131.

32. Bavinton BR, Brown G, Hurley M, et al. Which gay men would increase their frequency of HIV testing with home self-testing? AIDS Behav. 2013;17(6):2084-2092.

33. Nour S, Hsieh YH, Rothman RE, et al. Patients Can Accurately Perform Their Own Rapid HIV Point-of-Care Test in the Emergency Department. Point Care. 2012;11(4):176-179.

34. Gaydos CA, Solis M, Hsieh YH, Jett-Goheen M, Nour S, Rothman RE. Use of tablet-based kiosks in the emergency department to guide patient HIV self-testing with a point-of-care oral fluid test. Int J STD AIDS. 2013;24(9):716-721.

35. Gaydos CA, Hsieh YH, Harvey L, et al. Will patients "opt in" to perform their own rapid HIV test in the emergency department? Ann Emerg Med. 2011;58(1 Suppl 1):S74-S78. 
36. Kebede B, Abate T, Mekonnen D. HIV self-testing practices among Health Care Workers: feasibility and options for accelerating HIV testing services in Ethiopia. Pan Afr Med J. 2013;15:50.

37. Pant Pai N, Behlim T, Abrahams L, et al. Will an unsupervised selftesting strategy for HIV work in health care workers of South Africa? A cross sectional pilot feasibility study. PLoS One. 2013;8(11): e79772.

38. Frasca T, Balan I, Ibitoye M, Valladares J, Dolezal C, Carballo-DiéguezA. Attitude and behavior changes among gay and bisexual men after use of rapid home HIV tests to screen sexual partners. AIDS Behav. 2014;18(5):950-957.

39. Balán IC, Carballo-Diéguez A, Frasca T, Dolezal C, Ibitoye M. The impact of rapid HIV home test use with sexual partners on subsequent sexual behavior among men who have sex with men. AIDS Behav. 2014;18(2):254-262.

40. Carballo-Diéguez A, Frasca T, Balan I, Ibitoye M, Dolezal C. Use of a rapid HIV home test prevents HIV exposure in a high risk sample of men who have sex with men. AIDS Behav. 2012;16(7):1753-1760.

41. Carballo-Diéguez A, Frasca T, Dolezal C, Balan I. Will gay and bisexually active men at high risk of infection use over-the-counter rapid HIV tests to screen sexual partners? J Sex Res. 2012;49(4):379-387.

42. Golden MR, Stekler J, Hughes JP, Wood RW. HIV serosorting in men who have sex with men: is it safe? J Acquir Immune Defic Syndr. 2008;49(2):212-218.

43. Golden MR, Dombrowski JC, Kerani RP, Stekler JD. Failure of serosorting to protect African American men who have sex with men from HIV infection. Sex Transm Dis. 2012;39(9):659-664.

44. Philip SS, Yu X, Donnell D, Vittinghoff E, Buchbinder S. Serosorting is associated with a decreased risk of HIV seroconversion in the EXPLORE Study Cohort. PLoS One. 2010;5(9).

45. Wilson DP, Regan DG, Heymer KJ, Jin F, Prestage GP, Grulich AE. Serosorting may increase the risk of HIV acquisition among men who have sex with men. Sex Transm Dis. 2010;37(1):13-17.

46. van den Boom W, Konings R, Davidovich U, Sandfort T, Prins M, Stolte IG. Is serosorting effective in reducing the risk of HIV infection among men who have sex with men with casual sex partners? J Acquir Immune Defic Syndr. 2014;65(3):375-379.

47. Rosales-Statkus ME, Belza-Egozcue MJ, Fernández-Balbuena S, Hoyos J, Ruiz-García M, de la Fuente L. Who and how many of the potential users would be willing to pay the current or a lower price of the HIV self-test? The opinion of participants in a feasibility study of HIV selftesting in Spain. Enferm Infecc Microbiol Clin. 2014;32(5):302-305.

48. Lee SJ, Brooks R, Bolan RK, Flynn R. Assessing willingness to test for HIV among men who have sex with men using conjoint analysis, evidence for uptake of the FDA-approved at-home HIV test. AIDS Care. 2013;25(12):1592-1598.

49. Nunn A, Chan P, Towey C, Poceta J, Feller SC, Trooksin SB. Acceptability and Affordability of Self HIV Testing in an Urban Neighborhood with 3\% Seroprevalence. In: Programs and abstracts of the 21st Conference on Retroviruses and Opportunistic Infections (CROI); March 3-6, 2014; Boston, MA. Abstract 971.

50. Myers JE, El-Sadr Davis O, Weinstein ER, et al. Availability, Accessibility, Price of Rapid HIV Self-Tests, New York City Pharmacies, Summer 2013. In: Program and abstracts of the 21st Conference on Retroviruses and Opportunistic Infections (CROI); March 3-6, 2014; Boston, MA. Abstract 970.

51. Katz DA, Cassels SL, Stekler JD. Replacing clinic-based tests with home-use tests may increase HIV prevalence among Seattle men who have sex with men: evidence from a mathematical model. Sex Transm Dis. 2014;41(1):2-9.

52. Ibitoye M, Frasca T, Giguere R, Carballo-Diéguez A. Home testing past, present and future: lessons learned and implications for HIV home tests. AIDS Behav. 2014;18(5):933-949.
53. Karcher HL. HIV home test kit banned in Germany. BMJ. 1997; 315(7109):627.

54. Wright AA, Katz IT. Home testing for HIV. N Engl J Med. 2006;354(5): $437-440$.

55. Katz DA, Golden MR, Stekler JD. Use of a home-use test to diagnose HIV infection in a sex partner: a case report. BMC Res Notes. 2012;5:440

56. Labacher L, Mitchell C. Talk or text to tell? How young adults in Canada and South Africa prefer to receive STI results, counseling, and treatment updates in a wireless world. J Health Commun. 2013;18(12): 1465-1476.

57. Cabié A, Bissuel F, Huc P, Paturel L, Abel S. Impact of rapid HIV testing on the return rate for routine test results in sexually transmitted infection testing centres. Int J STD AIDS. 2011;22(12):757-758.

58. Choko AT, MacPherson P, Webb EL, et al. One year outcomes following community HIV self-testing: a prospective study in Malawi. In: Programs and abstracts of the 21 st Conference on Retroviruses and Opportunistic Infections (CROI). March 3-6, 2014; Boston, MA. Abstract 147.

59. US Food and Drug Administration. FDA approves first rapid diagnostic test to detect both HIV-1 antigen and HIV-1/2 antibodies [press release]. Silver Spring, MD: US Food and Drug Administration; August 8, 2013. Available from: http://www.fda.gov/newsevents/ newsroom/pressannouncements/ucm 364480.htm. Accessed February 24, 2014.

60. Masciotra S, Smith AJ, Youngpairoj AS, et al. Evaluation of the CDC proposed laboratory HIV testing algorithm among men who have sex with men (MSM) from five US metropolitan statistical areas using specimens collected in 2011. J Clin Virol. 2013;58 Suppl 1:e8-e12.

61. de la Fuente L, Rosales-Statkus ME, Hoyos J, et al; Madrid Rapid HIV Testing Group. Are participants in a street-based HIV testing program able to perform their own rapid test and interpret the results? PLoS One. 2012;7(10):e46555.

62. Masciotra S, Luo W, Youngpairoj AS, et al. Performance of the Alere Determine $^{\mathrm{TM}} \mathrm{HIV}-1 / 2 \mathrm{Ag} / \mathrm{Ab}$ Combo Rapid Test with specimens from HIV-1 seroconverters from the US and HIV-2 infected individuals from Ivory Coast. J Clin Virol. 2013;58 Suppl 1:e54-e58.

63. NASDAQ OMX GlobeNewswire. Chembio's SURE CHECK(R) HIV 1/2 Assay for Rapid Point-of-Care Detection of HIV Receives CE Mark Approval [press release]. Medford, NY: NASDAQ OMX GlobeNewswire; June 18, 2013. Available from: http://globenewswire.com/news-rele ase/2013/06/18/554787/10036662/en/Chembio-s-SURE-CHECK-RHIV-1-2-Assay-for-Rapid-Point-of-Care-Detection-of-HIV-ReceivesCE-Mark-Approval.html. Accessed March 21, 2014.

64. Yahoo! Finance Marketwire. Chembio Initiates Pre-IDE Studies for Home Use of Sure Check(R) Rapid HIV Test [press release]. Medford, NY: Yahoo! Finance Marketwire; June 27, 2011. Available from: http:// finance.yahoo.com/news/Chembio-Initiates-PreIDE-iw-3387913671. html. Accessed March 21, 2014.

65. Marlin R, Young S, Wilson G, et al. Feasibility of HIV self-test vouchers to raise community-level serostatus awareness, Los Angeles. In: Program and abstracts of the 21 st Conference on Retroviruses and Opportunistic Infections (CROI); March 3-6, 2014; Boston, MA. Abstract 969.

66. Young SD, Klausner J, Flynn R, Bolan R. Electronic vending machines for dispensing rapid HIV self-testing kits: a case study. AIDS Care. 2014;26(2):267-269.

67. Bristow CC, Adu-Sarkodie Y, Ondondo RO, et al. Standard Diagnostics Bioline HIV/Syphilis Duo Test: Multi-Site Laboratory Evaluation. In: Programs and abstracts of the 21st Conference on Retroviruses and Opportunistic Infections (CROI); March 3-6, 2014; Boston, MA. Abstract 627. 
HIV/AIDS - Research and Palliative Care

\section{Dovepress}

\section{Publish your work in this journal}

HIV/AIDS - Research and Palliative Care is an international, peerreviewed open-access journal focusing on advances in research in HIV, its clinical progression and management options including antiviral treatment, palliative care and public healthcare policies to control viral spread. The journal welcomes original research, basic science,

clinical \& epidemiological studies, reviews \& evaluations, expert opinion \& commentary, case reports \& extended reports. The manuscript management system is completely online and includes a very quick and fair peer-review system. Visit http://www.dovepress.com/ testimonials.php to read real quotes from published authors.

Submit your manuscript here: http://www.dovepress.com/hivaids---research-and-palliative-care-journal 